\title{
Implementation of statistical process control methods as a way to reduce production costs and improve product quality
}

\author{
Sergey Egorov ${ }^{1, *}$, Alexey Kapitanov ${ }^{1}$, and Dmitriy Loktev ${ }^{2}$ \\ ${ }^{1}$ Moscow state university of technology "STANKIN", 127055 Moscow Vadkovskiy per., 1, Russia \\ ${ }^{2}$ Technopolice Group, 117218 Moscow Dm. Ulyanova street, 42/1, Russia
}

\begin{abstract}
The article analyzes various situations of building a quality assurance system at an enterprise. The article includes the analysis of influence of various factors on the decision-making on the principles of building a quality assurance system, the conclusion about the need to build the quality assurance system aimed not at preventing the assembly of parts with deviations but on creating a process producing parts without deviations. The stages of implementation of the statistical process control system are considered. Moreover, the article analyzes the characteristics of measuring methods and tools that can be included into the quality assurance system, and examines the various process states allowing making a conclusion about the possibility of their controllability. The example of a comprehensive solution of the statistical process control system is given.
\end{abstract}

\section{Introduction}

During the lunch of any product, the quality requirements (as opposed to the production process requirements) are determined at the product design stage and cannot be changed at the manufacturing stage. A designer determines the main parameters of a finished product. These requirements are almost always determined by the market conditions - a consumer, on the one hand, and competitors, on the other hand. In other words, a product should completely meet the consumer's needs and at the same time be not worse and, most importantly, cheaper, than a competitor's product. Tightening of market competition results in constant tightening of the product requirements. After determining the requirements to a finished product, the designer determines the requirements to the parameters of individual parts. These requirements are converted into tolerance zones for certain sizes. By that, the designer proceeds from the premise that the technological process necessarily implements the tolerance zones assigned to it, and a product will have the planned parameters. The tolerance zone assigned by the designer is determined only by possible changes in the product parameters and very seldom takes into account possible difficulties in manufacturing of parts.

\section{Problem statement - ideal and realistic approach to technological processes}

After the designing stage, it is necessary to develop the technological process for individual parts. Here the first difficulties appear. The production engineer planning the technological process tries to select equipment and process parameters based on the design requirements.

Fine tolerances (determined in accordance with the market requirements) may not meet the equipment capabilities or may require the use of modern and more accurate tools. In any case, there is almost always a need to purchase new equipment and tools. But the production engineer is always sure that these investment needs will not be met. This can be partly explained with the lack of current assets (because a new product is only put on production and so far, it does not bring any money, hence the financing of manufacture of this new product should come from the funds earned on other projects or received from investors). Another reason may be the restriction concerning the product cost. As it has been already mentioned above, there are always market restrictions concerning the cost of a finished product. Taking into the account the project planned profitability, we determine the possible level of product cost and, as a consequence, the ultimate level of costs. In many cases, the calculation shows that implementation of the requirements specified by the designer without serious investments and, accordingly, without exceeding the planned level of costs is impossible. And in this case, the production engineer has to strike a compromise with the designer or to solve the problem using the methods not described in this article $[1,5]$.

Therefore, the production engineer was able to develop the technology meeting the necessary product requirements. The process of implementation of this technology starts. The parts are manufactured, inspected and shipped to a consumer as part of the product. New problems can appear at this stage again. It may be found

\footnotetext{
Corresponding author: egorovsergey@yandex.ru
} 
out that the parts do not correspond to the established tolerances. It may be found out at the stage of technical control and then the necessary measures are taken within the production. The worst situation is to receive a customer's complaint. This means that the inconsistency with the tolerances has been identified in the product in general and has resulted in the mismatch of the parameters of a finished product with the agreed specification.

In any of these "negative" scenarios, there is the analysis stage, the implementation of appropriate changes in technology, but the result is often similar to the one discussed above. The most unpleasant thing is that the inconsistency with the tolerances of individual parts or the inconsistency of the entire product with the agreed specification is not found out always, but at random times. The management natural reaction is toughening of the technical control requirements.

And here the manufacturer stands on the proven path - to ensure the product quality due to $100 \%$ verification of manufactured parts. This method of quality assurance has surely the right to exist, but it is fundamentally different from the methods used by the global leading companies for last two or three decades.

\section{Problem analysis}

Quality assurance due to $100 \%$ control is a traditional method, which is still used at many industrial enterprises. When using this method (Fig. 1), the problem of quality assurance is solved quite simply - it is necessary to separate good parts from bad ones. Moreover, it does not matter where (on what operation) and for what reason such defective product was manufactured. It is only important to detect it in time.

Good parts are assembled and shipped to customers, but bad parts are further analyzed to determine whether it is possible to make good parts from them (recoverable defect) or not (irrecoverable defect).

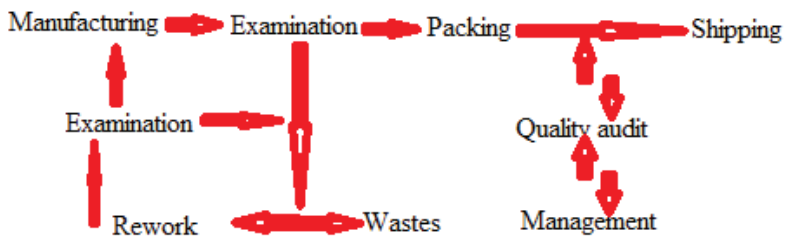

Fig. 1. Quality assurance due to $100 \%$ control.

It is quite obvious that the more parts are manufactured and the more complex these parts are (the more features should be controlled), the more controllers are required to check these parts. It is also obvious that the detected defects should be recovered - such parts should be completed (if it is a recoverable defect) or new parts should be manufactured instead of products with irrecoverable defects. The need to recover defects results, on the one hand, in increase in the production plan, and, on the other hand, in increase in production costs. The increase in the production plan cannot be eternal. If there are reserves of equipment performance, they are used to correct recoverable and irrecoverable defects. If the performance of processes is not enough, they start to assemble parts that are on the boundary between good and defective ones. If these parts are not enough, the production refers to the designer to get his/her permission to manufacture products with deviations. The designer has to expand the tolerances for the manufactured parts, and the previously defective products start to be assembled, but it helps to solve the performance problem. In some time, the quality of parts can improve, and the old design tolerances can be returned. Then the situation may become worse and everything may happen again.

The application of $100 \%$ control methods significantly increases the production costs. As it has been already shown above, if the problem of only determining defective products is solved, but not of preventing of its receipt, then the costs are substantially increased due to the recovery of defects. However, in addition to this cost item, the production cost of a part increases significantly due to the expenses for measuring. If a part (for example, an engine block) has about 1,000 different parameters (features) to be controlled, and 300 such blocks are manufactured per day, the quality assurance group should measure and make decisions on the compliance of 300,000 parameters per day. Such number of measurements requires an appropriate number of personnel and appropriate number of measuring tools that significantly increases the production costs and reduces the possible profit of this enterprise.

The necessity of total inspection of products is caused by the fact that the technological process, as a rule, does not always produce defective products. The situation, when the process produces only the defective products won't be considered here. This is related either to the incorrect tolerances, or to the complete unfitness of the process for manufacture of such products. Such situations are quite rare. In other cases, the process produces good products for some time, then defective ones, then again good ones, then the products, which are on the border between good and defective, and so on. The quality assurance system makes different decisions at different times, either accepting products, or directing them to be completed, or referring to the designer to get his/her permission.

With using this traditional approach, the management of an enterprise has to constantly make decisions related to the need to respond to the lack of suitable parts and incur additional costs, which are sometimes significant, to control products and correct defects.

The modern quality assurance system is aimed not at preventing the assembly of parts with deviations, but at creating a process that would produce parts without any deviations. In fact, if a process produces suitable parts at certain intervals, then it starts producing parts with deviations, then again suitable ones, and such transition occurs repeatedly, it means that there are some causes of that. It can be concluded that if these causes are found and eliminated, then the process will always produce only suitable products $[2,3,4]$.

Such modern approach is fundamentally different from the abovementioned traditional quality assurance 
approach due to $100 \%$ control of products. It also requires new attitude of enterprise management and production to quality assurance issues. To implement this approach, it is necessary to collect data on product parameters for a certain period of time, analyze them, find sources of changes and eliminate them. Then it is necessary to maintain the process monitoring the product parameters from time to time. If the process remains stable, you can be sure that only suitable parts will be assembled. The solution of the product quality issue will result in significant profit increase due to reduction of costs for $100 \%$ technical control, completion of recoverable defects and production of additional parts instead of irrecoverable defects. At the same time, the implementation of this new method will require certain costs, which are sometimes significant, which are quickly compensated due to increased profit.

It is possible to implement the analysis of existing problems affecting the quality of products, using the mathematical statistics methods. In this case they speak about the use of statistical process control. The concept "statistical process control" is defined as the use of statistical methods to achieve the quality of products or services by protecting the process from undesirable changes.

Statistical process control is related to mathematical statistics.

\section{Implementation of the statistical process control system}

Any process in nature is characterized by variability. It is affected by a huge number of different factors that change the process itself and its result. Considering cutting, we can find dozens of factors affecting the process. For example, hardness of a workpiece is not a constant value and even the hardness of workpieces from the best suppliers varies in the range of $10 \ldots 30 \mathrm{HB}$ units. Even positional repeatability of the best machine is no better than $1 . .5 \mu \mathrm{m}$. And so on. It means that changing of these factors will inevitably lead to the changes in parameters of the processed parts.

However, all these factors affect the process to a very small extent and, in most cases; it is very difficult to single out the impact of one of these factors. Such causes of process changing are called ordinary. The process remains stable due to the fact that there are many ordinary causes and each of them has its small effect, which is often compensated by other ordinary causes. From a statistical point of view, a stable process has constant distribution (Fig. 2 on the left).

If the process becomes instable, it is always associated with the appearance of specific causes or with a change in the set of ordinary causes. The causes of process changing that can be detected and identified are called specific. Any specific cause may lead to process instability. Then from a statistical point of view the process has inconstant distribution (Fig. 2 on the right).

In this case, the task of statistical process control is detection and elimination of specific causes of changing of product parameters, leading to the appearance of a product that does not meet the specified parameters.

The implementation of statistical process control system is carried out in several stages.

Statistical process control is based on analysis and interpretation of the initial data obtained from various sources. If the product geometrical parameters are evaluated, the initial data is obtained from various measuring tools. Besides, discrete features with two states (for example, presence or absence of a defect) may also be used.

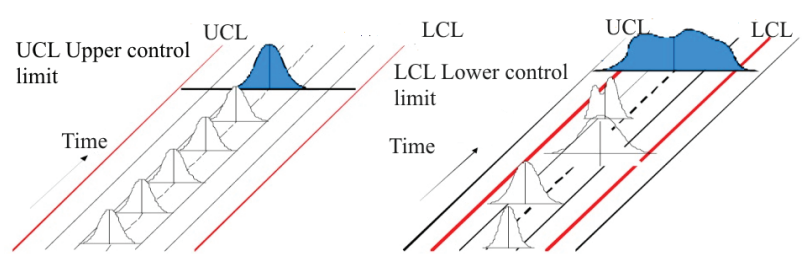

Fig. 2. Stable and instable process.

Therefore, at first it is necessary to determine a set of features for control of suitability of a part. Such features are dimensions and parameters of a part with certain tolerances. Depending on the role of controlled features in functioning of a finished product, they are divided into critical, very significant, significant and insignificant. Depending on the type of a feature, it is possible to determine different requirements to the stability of its implementation.

Various features (quality parameters) appear at different stages of the technological process. Therefore, at the second stage of the implementation of the statistical process control system, it is necessary to determine where, how and when the selected parameters will be measured. "Where" means both technological process operation and the organization of a measuring point. "How" implies the use of necessary measuring tools (more on this later) and the implementation of the measurement process itself. "When" in this case refers to determining a sample volume of parts for measuring and the measurement time interval of a given sample.

When choosing a measuring point and measuring tools, it is necessary to take into account the need to ensure reliability of the data send to the statistical analysis system. Data reliability is related to two main factors - unambiguousness of the obtained data and independence of data transmission from subjective factors. The first factor is associated with the use of certain measuring tools. To date, the use of digital measuring tools is an operational requirement to the implementation of the statistical process control system.

Indeed, it is difficult to talk about data reliability when reading information from a vernier of a conventional measuring tool because each operator sees slightly different values, and it calls into question unambiguousness of the obtained data. The second factor is also associated with the use of digital measuring tools and requires a direct connection of a measuring tool with the system for fixing the transmitted values. Human intervention in this process (an operator takes readings from a measuring tool and then manually enters them 
into the system for fixing values) results in the risk of deliberate or accidental data distortion [6,7].

Concluding the brief description of this stage of implementation of the process control system, we note that for further analysis of process controllability and for reflecting current statistics, the transmitted data should be accompanied by the full information on a product being measured and process parameters. Only in this case it is possible to conduct an effective analysis of the arising specific causes.

After determining parameters and methods for their measuring, it is required to make sure that the used measuring tools allow objectively assessing the quality of the selected parameters. In other words, a measuring tool should be suitable for measuring this value. This is not a matter of physical fitness (for example, inability to measure roughness parameters using the tools for measuring linear dimensions), but it is a matter of correspondence between the maximum error and the uncertainty of measurements of necessary accuracy associated with the tolerance zone of the measured parameter. There are various norms determining the ratio between the tolerance zone of the measured parameter and the maximum error of measuring tools.

This is always the first step in determining the suitability of a measuring tool. If the maximum error of a measuring tool exceeds 30\% (GOST requirement) of the tolerance zone of the measured parameter, then such measuring tool will be considered not suitable in almost all cases. In addition, measuring resolution must not exceed $5 \%$ of the tolerance zone of the measured parameter. If these two requirements are met, then the measurement system suitability is checked under realistic measurement conditions with the participation of real controllers. Various methods are practically used to assess the suitability of measuring tools. They determine the suitability indices $\mathrm{Cg}$ and $\mathrm{Cgk}, \mathrm{G} R \& R$ parameters (convergence and reproducibility) as well as some other parameters of suitability of measuring tools.

The whole complex of assessing measuring tools is standardized and included into the field of statistical methods. The European automotive industry uses VDA 5 standard, the American automotive industry uses the set of standards called MSA (Measuring System Analysis). The ISO standard regulating the suitability of measuring tools has recently appeared - ISO 22514-7:2012 [8,9].

After the parameters under evaluation are determined, the suitability of appropriate measuring tools is checked, they proceed to the next stage of the implementation of process control system - the check of equipment suitability for manufacturing of parts of certain precision.

To do this, at first, one part is processed on the used equipment. Based on the measurement results, appropriate corrections are made to obtain parameters in the middle of the given tolerance zone. Then five parts are processed successively, the selected parameters are fully measured and the spread of the obtained results is estimated. At this level, it is possible to make necessary corrections. For example, if the processing of five parts reveals a trend of resizing due to tool deterioration, it is possible to include appropriate software or hardware to eliminate this trend. And then fifty parts are processed on the machine. Based on the results of this processing, short-term reproducibility indices can be determined. Since the sample is small, international standards set quite high requirements for this parameter. If these requirements are met, then we can say that this equipment is suitable for the implementation of the required tolerance zones of the processed product.

Thus, after verification of suitability of the used equipment and measuring tools, we can proceed directly to the implementation of process control.

We are only interested in two questions - whether the process is stable (controllable) and, if so, how stable is this controllable state. In other words, we are interested whether the process can produce suitable products after appropriate adjustment and how reliable it is. The process indices - indices of reproducibility and process suitability - are used to assess the ability of the process to remain controllable.

If the process is statistically controllable, the process indices can be used to predict the further process behavior and, as a consequence, the possible share of defective products. Depending on this information, it is possible to make decisions on periodicity of product control. However, process indices work effectively only if the process is statistically controllable, i.e. there are no specific causes of process variability. This fact should be confirmed.

Control charts are used to assess process stability and to identify the presence of specific causes. The theory and practice of using control charts were developed in the middle of 20th century and has been constantly improving since that moment. Two control charts are made (Fig. 3) - mean values and standard deviations (or swings) - to control the process. The first chart allows assessing stability of the mean value position of the observed parameter. The second chart shows dispersion (spread) of the values of the considered parameter. Control charts should be used only in pairs, otherwise there is a risk of making a false decision.

At the beginning of using control charts, all necessary calculations were made manually. Although control charts are based on the serious theory of mathematical statistics, the calculations are quite simple. With the development of computer technology, the calculation of control charts was fully automated.

When creating each control chart, first the values of upper and lower control limits are calculated based on the data of the measured value. Then, the current state of the process is assessed to sample the data, and it is recorded as a point on the control chart. The main sign of process instability is location of one of the points beyond the control limits (see Fig. 3, marked in red). There are other criteria for assessing process controllability in accordance with control charts (certain trends of the location of points).

If a point is located beyond the control limits, the responsible personnel decide on the need to adjust the process. They identify a specific cause, which has affected the process stability, and take measures to eliminate the damage from this specific cause. After that, 
the process is further controlled until the control charts show full controllability of the process.

Control charts allow qualitatively assessing the presence of specific causes and effectiveness of the measures taken to eliminate them. If a control chart shows that the process is statistically controlled (no process control criterion is violated), then it is possible to assess how stable the process is.

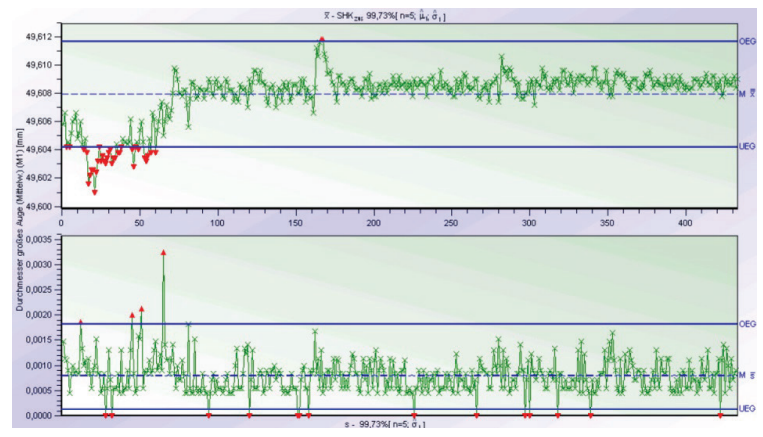

Fig. 3. Example of control charts.

Special indices as the indices of reproducibility and process suitability are used to quantify the process stability. The reproducibility index $C_{p}$ shows the ratio of permissible process spread (i.e., width of the tolerance zone) to actual process spread (Fig. 4). The value $C_{p}=1$ corresponds to the process reproducibility limit (it corresponds to the control limits on the control charts). In the absolute majority of cases, the minimum value of process reproducibility is more than 1 (usually 1.33). In this case, the actual process spread should not exceed $75 \%$ of the tolerance zone, and the process guarantees to obtain no more than 84 defective parts per million of manufactured parts.

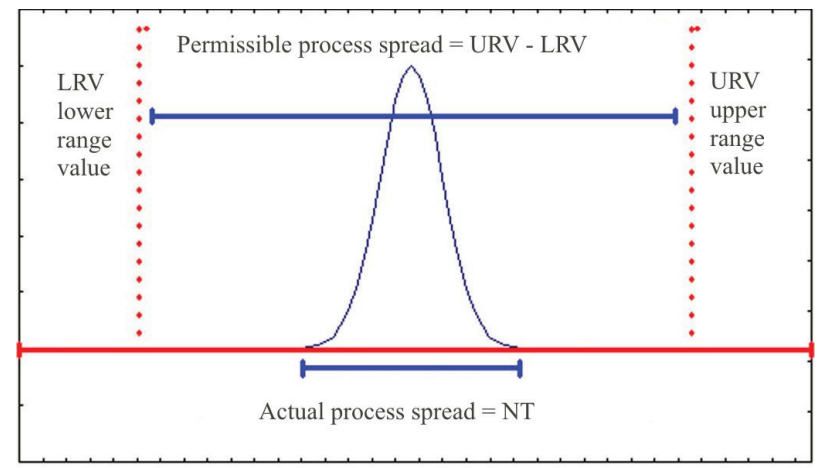

Fig. 4. Ratio of permissible process spread to actual one.

The reproducibility index $\mathrm{C}_{\mathrm{p}}$ reflects the process potential, but it does not take into account the location of actual spread limits relative to the nominal value of the considered value. The reproducibility index $\mathrm{C}_{\mathrm{pp}}$ is used to consider the process centering. This index assesses the distance between the mean process value and the nearest tolerance zone boundary. The higher this index is, the better the process is centered [10,11]. Absolutely centered processes have similar $\mathrm{C}_{\mathrm{p}}$ and $\mathrm{C}_{\mathrm{pk}}$.

In certain cases, the index of process suitability $\mathrm{P}_{\mathrm{p}}$ and $\mathrm{P}_{\mathrm{p \kappa}}$ are used instead of the reproducibility index $\mathrm{C}_{\mathrm{p}}$ and $\mathrm{C}_{\mathrm{p \kappa}}$. The difference between these groups of indices is the method of assessing actual process spread. In general, all four indices are united with a common name of the process capability values.

Thus, the combination of control chart analysis and calculation of process capability values makes it possible to determine qualitatively and quantitatively whether the process is in a statistically controllable state. If not, there is a certain algorithm of actions and certain tools to bring the process to a controlled state. Without going into details, we can mention such tools as a cause and effect diagram (Ishikawa chart/fishbone graph) and a Pareto chart.

\section{Comprehensive solution conclusions and perspectives}

One may feel that the implementation of statistical process control requires significant costs, both mental and material. In fact, it is not so. With the help of qualified consultants, the statistical process control system may be implemented in a short period of time with optimal investments.

To do this, you need to meet only two basic conditions - to equip the production with modern measuring tools, which reliably allow obtaining and transmitting process data, and to implement the control system itself. It can be easily done with the help of specialized software. One of the most famous brands in this field is Q-DAS (Germany), which offers a full set of software for the implementation of statistical process control and is a world leader in this direction.

The concept of statistical process control software product based on modern measuring tools is called CAMERA and implemented at six levels (Fig. 5).

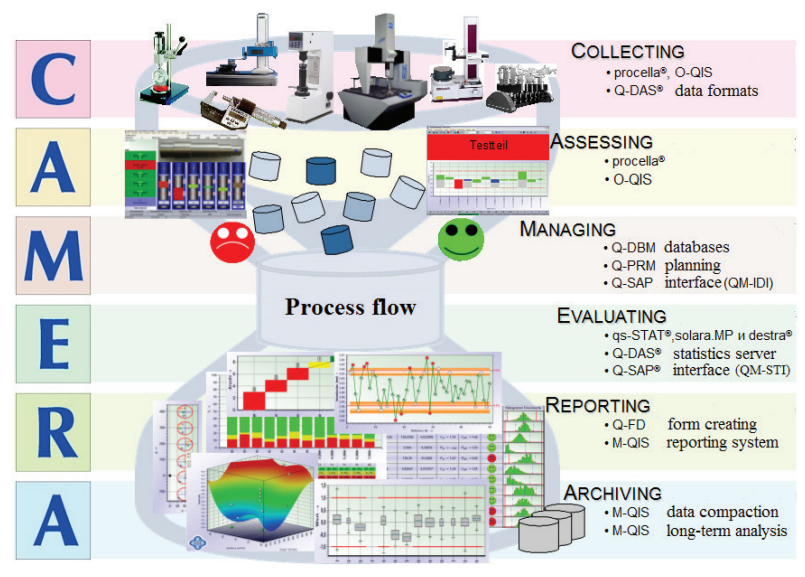

Fig. 5. Concept of CAMERA software product.

The first level includes the collection of data using various measuring tools suitable for measuring the considered parameters and creating process parameter values in sufficient volume and with sufficient reliability. Specialized software products (procella My.SPC and O-QIS) are used to connect the measuring tools with the systems for assessing process parameters and to obtain data in the required format. It should be noted that the data formats created by Q-DAS are supported by almost all suppliers of measuring systems. 
The second level includes primary assessment of the obtained data using the same software products. The assessment results in getting the process parameters process flow, histograms, control charts, indices of reproducibility and suitability, etc. These assessment results can be presented in various forms depending on their recipient [12-15].

At the third level the data are transmitted to the central database.

The fourth level includes a deeper analysis of the obtained data, if necessary. Solara.MP software implements the analysis of suitability of measuring tools. The software product qs-STAT is designed to obtain almost any statistical process evaluations, and destra software allows optimizing the studied process using statistical methods (for example, regression and variational analysis)

The fifth level includes reporting on the conducted evaluations. Reporting forms and content can be changed depending on their recipient.

Finally, the sixth level includes archiving of the received data for its further storage and long-term analysis.

The research is performed with financial support of the Russian Ministry of Education and Science (project № 11.1817.2017/4.6).

\section{References}

1. D. Loktev, S. Egorov, A. Kapitanov, V. Mitrofanov, and T. Egorova, Russian Engineering Research Vol. 36, No. 4, pp. 312-317 (2016)

2. Sergey B. Egorov, Alexey V. Kapitanov, Vladimir G. Mitrofanov, Leonid E. Shvartsburg, Natalia A. Ivanova, and Sergey A. Ryabov, IEJME Mathematics Education Vol. 11, No. 7, pp. 22132225 (2016)

3. S. Egorov, A. Kapitanov, D. Loktev, T. Egorova International journal of experimental education № 10-1, pp. 127-129 (2016)

4. D. Loktev, S. Egorov, A. Kapitanov, V. Mitrofanov, T. Egorova, STIN № 9, pp. 13-19 (2015)

5. S. Egorov, A. Kapitanov, T. Egorova, V. Mitrofanov, International journal of experimental education № 12-1, pp. 128-129 (2015)

6. S. Egorov, A. Kapitanov, T. Egorova, D. Loktev, V. Mitrofanov, Innovation's №08 (202), pp. 85-90 (2015)

7. D. Loktev, S. Egorov, A. Kapitanov, A. Loktev, V. Mitrofanov, T. Egorova. Materials of the VI International Scientific Conference, Vol. II, p. 276 (2015)

8. S. Egorov, D. Loktev, A. Kapitanov, V. Mitrofanov, T. Egorova, Eurasian Union of scientists №5, pp. 51-54 (2015)

9. S. Egorov, A. Kapitanov, D. Loktev, A. Loktev, V. Mitrofanov, Vestnik MSTU «Stankin» №4(35), pp. 83-88 (2015)
10. S. Egorov, A. Kapitanov, V. Mitrofanov, T. Egorova, WCSE (2016).

11. Sergey B. Egorov, Alexey V. Kapitanov, Vladimir G. Mitrofanov, Leonid E. Shvartsburg, Natalia A. Ivanova and Sergey A. Ryabov, International journal of environmental \& science education Vol. 11, No. 11, pp. 4065-4078 (2016)

12. S. Egorov, A. Kapitanov, D. Loktev, Materials Science Forum, Vol. 870, pp. 397-403, (2016)

13. S. Egorov, A. Kapitanov, D. Loktev, II International scientific and technical conference "Industrial Engineering" (2016).

14. D. Loktev, S. Egorov, A. Kapitanov, V. Mitrofanov, T. Egorova, Innovation's 08(214), p. 113-119 (2016)

15. D. Loktev, S. Egorov, A. Kapitanov, V. Mitrofanov, T. Egorova, Problems of mechanical engineering and automation №3, p. 110-119 (2015)

16. S. Egorov, Fundamental research №6, vol. 5, pp. 920-927 (2014)

17. S. Egorov, Fundamental research №8, vol. 1, pp. 2631 (2014) 